\title{
EOS Imaging System is Available for Early Onset Scoliosis and Can Reduce Ionizing Radiation Exposure
}

\author{
Burt Yaszay ${ }^{1,}{ }^{,}$, Nima Kabirian ${ }^{2}$, Gregory M. Mundis ${ }^{3}$, Carrie E. Bartley ${ }^{1}$, Jeff Pawelek ${ }^{2}$ and Behrooz A. \\ Akbarnia $^{4}$ \\ ${ }^{1}$ Rady Children's Hospital, San Diego, California, United States \\ ${ }^{2}$ Growing Spine Foundation, Milwaukee, Wisconsin, United States \\ ${ }^{3}$ Scripps Clinic Torrey Pines, La Jolla, California, United States \\ ${ }^{4}$ University of California, San Diego, California, United States \\ ‘Corresponding author: Rady Children's Hospital, San Diego, California, United States. Email: byaszay@rchsd.org
}

Received 2019 March 23; Accepted 2019 April 26.

\begin{abstract}
Background: Long-term ionizing radiation exposure is a risk to young scoliosis patients. A new slot-scanning imaging system, "EOS imaging" has been shown, in patients with adolescent idiopathic scoliosis, to emit significantly lower levels of ionizing radiation. This is a single-center, preliminary report of radiographic assessment by a new system.

Methods: Thirty-eight patients with early onset scoliosis (EOS) who had spine radiographs (antero-posterior, lateral or bending) were included. Patients were divided into two groups: (1) conventional radiography (CR) and (2) EOS-imaging. Patients's demographics and total annual radiation (TAR) doses were calculated. The mean TAR dose per patient was compared to annual background radiation estimated by Radiological Society of North America $(2.4 \mathrm{mSv})$. The mean radiation dose for anteroposterior and lateral spine film with the EOS imaging system was reported as 0.12 and $0.19 \mathrm{mSv}$ per the manufacturer.

Results: There were 25 patients in the CR and 18 patients in the EOS-imaging group. Five patients had films from both CR and EOSimaging on different occasions. Mean follow-up of the entire cohort from the first spine x-ray was 1.3 years ( $0.3-2$ years). The mean TAR dose per patient was $10.2 \mathrm{mSv}(3.3-20.3)$ and $1.3(0.6$ - 2.2) for CR and EOS-imaging groups, respectively. The mean TAR was $4.25 \times$ $(\mathrm{CR})$ and $0.54 \times$ (EOS-imaging) that of annual background radiation. The mean age of patients at the first spine X-ray within the study period was 4.1 years ( 0.11 - 9.2) in CR and 7.6 year $(3.3-10.5)$ in the EOS-imaging group.

Conclusions: Our study shows the TAR dose per patient from EOS imaging systems are lower than conventional systems and can be utilized for patients as young as 3 years old. This study suggests that the use of this new system can reduce TAR dose in EOS patients; however, a larger cohort with longer follow-up is needed to critically examine this statement.
\end{abstract}

Keywords: EOS Imaging, Early Onset Scoliosis, Radiation

\section{Background}

Early onset scoliosis (EOS) is a uniquely challenging condition to treat. Numerous surgical and non-surgical treatment options exist including casting, bracing, growing rods, Shilla, vertical expandable prosthetic titanium rib (VEPTR), growth modulation, and limited fusion techniques (1-3). Because of the challenges presented by EOS and the associated risk of thoracic insufficiency and pulmonary compromise, early intervention is preferred. In many cases, multiple treatments may be employed (e.g. serial casting followed by growing rod placement) to control the deformity long enough to allow the spine and thoracic space to grow prior to a definitive "final" spinal fusion (4).

Regardless of the treatment, properly monitored EOS patients attend numerous doctor visits where they are ex- posed to ionizing radiation (IR) via repeated spine radiographs. Patients undergoing surgical treatment are often exposed to IR intraoperatively in addition to their clinical visits. It is not unreasonable for EOS patients and their families to endure several years of doctor visits and procedures, many of which require exposure to IR. Flynn et al. followed 99 patients through their entire course of growing rod treatment and reported that the average duration of treatment was $5.0 \pm 2.6$ years (5).

Cannon et al. evaluated IR exposure in EOS patients undergoing VEPTR treatment and found that over a fouryear period, there was an average of five procedures per patient and an average of 40 imaging studies per patient, for a total of 962 images among 24 patients (6). Imaging studies included radiographs computed tomography, in- 
traoperative fluoroscopy, and nuclear medicine. Similarly, Khorsand et al. reported on IR exposure in 62 EOS patients and thoracic insufficiency syndrome who also underwent VEPTR treatment (7). There were a total of 4,293 radiographs taken with an average of 69 images per patient. Total IR exposure in this group, including computed tomography (CT), MRI, radiographs, and ventilation/perfusion scans, was estimated at four times the amount of the estimated annual background radiation exposure in the Unites States, with the majority of IR coming from CT scans. Additionally, Mundis et al. (8) reported EOS patients who underwent growing rod treatment were exposed to 3.4 times the IR exposure than estimated background radiation within the same time period. In their study, younger growing rod patients and those who underwent revision surgery were exposed to higher IR doses, with plain radiographs accounting for $89 \%$ of the total IR.

Techniques to reduce IR in this patient population are certainly needed. In recent years, a biplanar slot scanning X-ray system was introduced that simultaneously obtains anteroposterior and lateral radiographs and reportedly emits a fraction of the amount of IR than that produced by traditional, conventional radiography (CR) (9). This system, known as EOS imaging (EOS imaging, Paris, France), has been shown to reduce IR exposure in AIS patients by 6 to 9 times less than that of CR. The biplanar films allow for the possibility of 3D reconstructions of the spine to be created from 2D X-rays. Previous studies have reported the $3 \mathrm{D}$ reconstructions to be reliable and comparable to $\mathrm{CT}(10,11)$. In addition to reducing IR exposure, the EOS imaging system takes less time to obtain the two films than standard digital radiographs (12). Deschenes et al. evaluated image quality and radiation dose between $C R$ and EOS imaging images taken of the same patients (13). They found the EOS imaging quality was greater than that of the CR images, with the exception of the visualization of the spinous process on sagittal views. They also found IR exposure was reduced 6 to 9 times in the thoracolumbar region and 3 times in the neck region with the use of EOS imaging. The majority of these studies using EOS-images have focused on adolescents, however, with little to no emphasis on the EOS population.

\section{Objectives}

The purpose of the current study was to evaluate differences in IR exposure between CR and EOS-images of the spine in patients with EOS who have been treated with growing rod constructs.

\section{Methods}

IRB approval from the affiliated authors' institution was obtained. Thirty-eight EOS patients who had scoliosis radiographs (anteroposterior, lateral or bending) were included. All patients who had EOS imaging must have been able to stand still for a period of approximately 10 - 15 seconds. Patients who are non-ambulatory or able to stand still for the required period of time are not currently eligible for EOS imaging. Patient etiologic diagnoses included idiopathic (14), syndromic (12), congenital (9), and neuromuscular (7) based on the classification of EOS (14). All patients were surgically treated surgically. Patients were divided into two groups: (1) Conventional radiography group (CR) and (2) EOS imaging group. Patients' demographics and total annual radiation (TAR) dose were calculated in millisieverts ( $\mathrm{mSv}$ ). The mean TAR dose per patient was compared to annual background radiation estimated by Radiological Society of North America (2.4 mSv per year). The mean radiation dose per single image for anteroposterior and lateral spine film with the EOS imaging system was reported as 0.12 and $0.19 \mathrm{mSv}$ per the manufacturer.

\section{Results}

There were 25 patients in the CR group and 18 patients in the EOS imaging group. Five patients had films from both CR and EOS imaging on different occasions. The mean age of patients at the first spine X-ray within the study period was 4.1 years ( 0.11 - 9.2) in CR group and 7.6 year (3.3 $10.5)$ in EOS imaging group. Mean follow-up of the entire cohort from the first spine X-ray was 1.3 years $(0.3-2)$. The mean TAR dose per patient was 10.2 $\mathrm{mSv}$ (range, 3.3 - 20.3 $\mathrm{mSv}$ ) and 1.3 (range, 0.6 - $2.2 \mathrm{mSv}$ ) for CR and EOS imaging groups, respectively, which is 4.25 times and 0.54 times that of the annual background radiation. The mean total radiation dose for each patient group broken down by etiologic categories is shown in Table 1 . The majority of the patients in the EOS imaging group were idiopathic (61\%) whereas patient etiology was more evenly distributed in the CR group.

\section{Discussion}

Primarily due to their young age, patients with EOS are expected to endure several years of repeated spine radiographs. This preliminary study on our series of EOS patients showed the TAR dose per patient from EOS imaging was lower than conventional X-ray and can be utilized for patients as young as 3 years old. 


\begin{tabular}{lccc}
\hline \multicolumn{1}{l}{ Table 1. The Mean Total Radiation Dose for Each Patient Group Broken down by Etiologic Categories } & \\
\hline & Idiopathic & Syndromic & Congenital \\
\hline Conventional X-ray & $13.38 \mathrm{mSv}(\mathrm{N}=4)$ & $12.1 \mathrm{mSv}(\mathrm{N}=8)$ & $8.42 \mathrm{mSv}(\mathrm{N}=7)$ \\
EOS imaging & $1.32 \mathrm{mSv}(\mathrm{N}=11)$ & $1.38 \mathrm{mSv}(\mathrm{N}=4)$ & $0.95 \mathrm{mSv}(\mathrm{N}=2)$ \\
\hline
\end{tabular}

The average TAR dose per patient in our study was 10.2 $\mathrm{mSv}$ for CR and $1.3 \mathrm{mSv}$ for EOS imaging. These results are similar to those found in previous studies that have reported significantly reduced IR exposure to patients using the biplanar slot scanning machine $(12,13)$. The mean TAR was 4.25 times that of annual background radiation in the CR group and 0.54 times greater in the EOS imaging group. Previous reports have also noted an increased amount of IR exposure in EOS patients ranging from 3.4 times (8) to 4 times (7) that of estimated annual background radiation. However, it should be noted that in our study, the increased TAR in relation to background radiation likely underestimates the total amount of IR exposure to this patient population as we did not include other forms of medical radiation in our analysis, such as CT scanning and non-spine IR medical imaging.

Other factors that have been previously shown to influence the amount of IR exposure in EOS patients include etiology, revision surgery, age of the patient, and surgeon experience $(6,8)$. Cannon et al. found that radiation exposure in EOS patients undergoing VEPTR treatment decreased as surgeon experience increased (6). Additionally, they found that congenital scoliosis patients, as opposed to neuromuscular scoliosis patients, received greater amounts of radiation. Similarly, Mundis et al. (8) reported that from initial spine film to one year after surgery, congenital scoliosis patients, followed by syndromic, received the greatest amount of IR. Our current study found that the mean TAR was highest in idiopathic scoliosis and syndromic scoliosis patients, although a statistical comparison between etiologies was not conducted due to the limited sample size and an etiologic comparison was beyond the scope of our study aim.

The majority of the patients (61\%) in the EOS imaging group had idiopathic scoliosis. The majority of the congenital, syndromic, and neuromuscular patients were in the $\mathrm{CR}$ group. The mean age of patients at initial imaging in the CR group was lower (4.1 years) than the EOS imaging group (7.6 years). We found that the EOS imaging system can be used successfully for patients as young as 3 years old. Because EOS imaging is performed via a scanning mechanism, patients who have difficulty remaining still for a period of 10 - 15 seconds may not be good candidates for these radiographs, as this will create motion artifact. However, our study shows EOS patients who are eligible for EOS imaging can expect a much lower dose of IR exposure compared to conventional X-ray systems. A larger cohort of EOS patients who have completed treatment will be needed to confirm these preliminary findings.

\section{Footnotes}

Authors' Contribution: All authors contributed in study design, data gathering, manuscript writing and revising.

Conflict of Interests: There are no direct conflicts of interests.

Ethical Considerations: IRB approved study.

Funding/Support: No funding was received for this study.

\section{References}

1. Ridderbusch K, Rupprecht M, Kunkel P, Stucker R. [Non-fusion techniques for treatment of pediatric scoliosis]. Orthopade. 2013;42(12):1030-7. German. doi: 10.1007/s00132-012-2049-x. [PubMed: 23982693].

2. Farrington DM, Tatay-Diaz A. [Early onset scoliosis. What are the options?]. Rev Esp Cir Ortop Traumatol. 2013;57(5):359-70. Spanish. doi: 10.1016/j.recot.2013.05.004. [PubMed: 24071039].

3. Fletcher ND, Bruce RW. Early onset scoliosis: Current concepts and controversies. Curr Rev Musculoskelet Med. 2012;5(2):102-10. doi: 10.1007/s12178-012-9116-0. [PubMed: 22477364]. [PubMed Central: PMC3535151].

4. Waldron SR, Poe-Kochert C, Son-Hing JP, Thompson GH. Early onset scoliosis: The value of serial risser casts. J Pediatr Orthop. 2013;33(8):775-80. doi: 10.1097/BPO.0000000000000072. [PubMed: 23965912].

5. Flynn JM, Tomlinson LA, Pawelek J, Thompson GH, McCarthy R, Akbarnia BA, et al. Growing-rod graduates: Lessons learned from ninetynine patients who completed lengthening. J Bone Joint Surg Am. 2013;95(19):1745-50. doi: 10.2106/JBJS.L.01386. [PubMed: 24088966].

6. Cannon TA, Astur Neto N, Kelly DM, Warner WC Jr, Sawyer JR. Characterization of radiation exposure in early-onset scoliosis patients treated with the vertical expandable prosthetic titanium rib. J Pediatr Orthop. 2014;34(2):179-84. doi: 10.1097/BPO.0000000000000079. [PubMed: 24172680].

7. Khorsand D, Song KM, Swanson J, Alessio A, Redding G, Waldhausen J. Iatrogenic radiation exposure to patients with early onset spine and chest wall deformities. Spine (Phila Pa 1976). 2013;38(17):E1108-14. doi: 10.1097/BRS.ob013e318299fb82. [PubMed: 23670344].

8. Mundis GMN, Hennessy MW, Pawelek J, Yaszay B, Akbarnia BA. Longitudinal analysis of radiation exposure during the course of growing rod treatment for early onset scoliosis. International Meeting on Advanced Spine Techniques. 2012.

9. Dubousset J, Charpak G, Dorion I, Skalli W, Lavaste F, Deguise J, et al. [A new 2D and 3D imaging approach to musculoskeletal physiology and 
pathology with low-dose radiation and the standing position: The EOS system]. Bull Acad Natl Med. 2005;189(2):287-97. discussion 297300. [PubMed: 16114859].

10. Glaser DA, Doan J, Newton PO. Comparison of 3-dimensional spinal reconstruction accuracy: Biplanar radiographs with EOS versus computed tomography. Spine (Phila Pa 1976). 2012;37(16):1391-7. doi: 10.1097/BRS.ob013e3182518a15. [PubMed: 22415001].

11. Al-Aubaidi Z, Lebel D, Oudjhane K, Zeller R. Three-dimensional imaging of the spine using the EOS system: Is it reliable? A comparative study using computed tomography imaging. J Pediatr Orthop B. 2013;22(5):409-12. doi: 10.1097/BPB.0b013e328361ae5b. [PubMed: 23652967].

12. Dietrich TJ, Pfirrmann CW, Schwab A, Pankalla K, Buck FM. Comparison of radiation dose, workflow, patient comfort and financial break- even of standard digital radiography and a novel biplanar low-dose X-ray system for upright full-length lower limb and whole spine radiography. Skeletal Radiol. 2013;42(7):959-67. doi: 10.1007/s00256-0131600-0. [PubMed: 23536038].

13. Deschenes S, Charron G, Beaudoin G, Labelle H, Dubois J, Miron MC, et al. Diagnostic imaging of spinal deformities: Reducing patients radiation dose with a new slot-scanning X-ray imager. Spine (Phila Pa 1976). 2010;35(9):989-94. doi:10.1097/BRS.0b013e3181bdcaa4. [PubMed: 20228703].

14. Williams BA, Betz RR, Blakemore LC, Flynn JM, Johnston CE, McCarthy $\mathrm{RE}$, et al. Introducing the early onset scoliosis classification system. International Meeting on Advanced Spinal Techniques (IMAST);. July 18-21. 2012 